Article

\title{
Improving Altimeter Wind Speed Retrievals Using Ocean Wave Parameters
}

\author{
Haoyu Jiang ${ }^{1,2,3^{*}}$, Hao Zheng ${ }^{1}$ and Lin Mu ${ }^{1,3 *}$ \\ 1 College of Marine Science and Technology, China University of Geosciences, Wuhan 430074, China; \\ Haoyujiang@cug.edu.cn; Cugwh521@foxmail.com; Moulin1977@hotmail.com; \\ 2 Laboratory for Regional Oceanography and Numerical Modeling, Qingdao National Laboratory for \\ Marine Science and Technology, Qingdao 266100, China \\ 3 Shenzhen Research Institute, China University of Geosciences, Shenzhen 518057, China \\ * Correspondence: Haoyujiang@cug.edu.cn; Tel.: +86-1895-421-5115 (H. J.), Moulin1977@hotmail.com; Tel: \\ +86-1350-209-8253 (L. M.)
}

\begin{abstract}
Spaceborne altimeters are an important data source for obtaining global sea surface wind speeds (U10). Although many altimeter U10 algorithms have been proposed and they perform well, there is still room for improvement. In this study, the data from ten altimeters were collocated with buoys to investigate the error of the altimeter U10 retrievals. The U10 residuals were found to be significantly dependent on many oceanic and atmospheric parameters. Because these oceanic and atmospheric parameters are inter-correlated, an asymptotic strategy was used to isolate the impact of different parameters and establish a neural-network-based correction model of altimeter U10. The results indicated that significant wave heights and mean wave periods are effective in correcting U10 retrievals, probably due to the tilting modulation of long-waves on the sea surface. After the wave correction, the root-mean-square error of the retrieved U10 was reduced from $1.42 \mathrm{~m} / \mathrm{s}$ to 1.24 $\mathrm{m} / \mathrm{s}$ and the impacts of thermodynamic parameters, such as sea surface (air) temperate, became negligible. The U10 residuals after correction showed that the atmospheric instability can lead to errors on extrapolated buoy U10. The buoy measurements with large air-sea temperature differences need to be excluded in the Cal/Val of remotely sensed U10.
\end{abstract}

Keywords: Altimeter, Sea Surface Wind Speed, Significant Wave Height, Mean Wave Period, Atmospheric Instability

\section{Introduction}

Sea surface wind speed is one of the key environmental parameters in marine and atmospheric sciences. Spaceborne active microwave remote sensors, including radar altimeter, scatterometer, and Synthetic Aperture Radar (SAR), can all be used to retrieve $10-\mathrm{m}$ sea surface wind speed (U10, henceforth) because the Radar Cross-Sections (RCSs) are sensitive to sea surface roughness (SSR) [1], and the SSR is closely correlated to U10. Among these remote sensors, scatterometers have the widest swath and the best overall accuracy (with a typical error of $\sim 1 \mathrm{~m} / \mathrm{s}$ ) [2], making them an irreplaceable data source of U10. Meanwhile, radar altimeters, with a typical error of wind speed of $1.5 \mathrm{~m} / \mathrm{s}$ (e.g., $[3,4])$, have a better accuracy in high wind speeds [1] and can provide global coverage of U10 and significant wave height $(\mathrm{SWH})$ data simultaneously, making them also a unique tool for studies in wind-waves (e.g., [5-7]). Since 1985, more than ten satellite altimeters have been launched, which provide sufficiently long data records for studies of wind and wave climate [5].

RCSs are related to U10 through the Geophysical Model Function (GMF). For altimeters, the GMF is based on the quasi-specular scattering: The SSR is believed to be dominantly controlled by $\mathrm{U} 10$, and a smoother sea surface corresponds to a larger RCS and vice versa. When establishing the retrieving algorithm, however, the RCSs are usually directly mapped to U10 obtained from other instruments, such as buoys or scatterometers [4]. Although the derived GMFs generally have a good 
performance in measuring U10 (e.g., [1,2,4]), it is noted that U10 is not the only impact factor for SSR. For example, surface ocean currents and atmospheric instability can all have a direct impact on the SSR response to U10 (e.g., [8,9]), thus impacting the wind retrieval. In addition, recent studies show that some parameters of the oceanic environment have significant impacts on the wind speed retrievals from C-band scatterometers and SARs with tilt incident angles (e.g., [10-12]). For example, Sea Surface Temperature (SST) can slightly change the SSR's response to U10 by impacting dynamic viscosity of water [11,13] and by impacting the atmospheric stability [12], and ocean swells can also change this response by impacting the tilt of sea surface [10,12]. Better accuracy of U10 may be achieved by considering these secondary responses.

Wind retrievals from altimeters may also be impacted by these effects. Among which, the impact of long-wave tilting of capillary waves has been well known (e.g., [14]) and SWH has been considered in some altimeter GMFs (e.g., [15]). Meanwhile, ocean waves may impact altimeter wind retrieval in many ways. For instance, wave crests are usually rougher than wave troughs, which is a very important effect for retrieving sea surface height termed electromagnetic bias (e.g., [16]). It may also impact U10 retrievals because the RCS is a "linear average" over the $\sim 7 \mathrm{~km}$ footprint for the $1-\mathrm{Hz}$ data. Other oceanic and atmospheric parameters such as wave periods, precipitation, SST, and Sea Surface Air Temperature (SSAT), might also impact altimeter U10 retrievals. This study aims to conduct a quantitative analysis of how much altimeter U10 retrievals are impacted by these parameters of the ocean environment, and a trial was made to improve the data accuracy by considering the impact of these parameters.

The remainder of this paper is organized as follows: The collocated dataset between altimeter and in-situ observations are described in Section 2. In Section 3, the differences between the altimeter and in-situ U10 data (residuals) are analyzed relative to some parameters of the ocean environment. A correction model for altimeter U10 retrievals based on the correlations in Section 3 is presented in Section 4. Conclusions are given in Section 5.

\section{Data and Methods}

\subsection{Multiplatform Altimeter Dataset}

Systematic calibrations and validations of simultaneous U10 and SWH data from 13 altimeter missions (GEOSAT, ERS-1/2, TOPEX, GFO, JASON-1/2/3, ENVISAT, CRYOSAT-2, HY-2, SARAL, and SENTINEL-3A) over 33 years (1985-2018) were conducted by [3] based on a previous work of [17]. Because of the joint calibration, the systematic errors of U10 and SWH between different altimeters were preliminarily eliminated. The data of the calibrated U10 from ten satellites (TOPEX, ERS-2, GFO, JASON-1/2/3, ENVISAT, JASON-2, Cryosat-2, and HY-2) over the period from 2001 to 2017 is selected in this study. The reason for using data from different altimeters is to obtain more collocations with buoys for better statistical significance. It was assumed that the impact of oceanic and atmospheric parameters induces a "systematic error" which is similar for all altimeters using the same frequency band, and thus the data from Ka-band SARAL are not used here. All the altimeter U10 data used in this study were retrieved from Ku-band RCS as reported in [3], and the information of SWH and RCS of other frequencies (C-band and S-band) were not taken into consideration. The data with bad quality flags or U10 $>25 \mathrm{~m} / \mathrm{s}$ or $\mathrm{SWH}>10 \mathrm{~m} / \mathrm{s}$ were discarded. More detailed information on this dataset is available in [3].

\subsection{Buoy Observations}

The quality controlled in-situ data from the National Data Buoy Center (NDBC) were used to assess the altimeter U10 retrievals. Fifty-two buoys more than $50 \mathrm{~km}$ offshore were used and the locations of these buoys are shown in Figure 1. Most anemometers of the NDBC buoys measure wind vectors at a height of 2-5 $\mathrm{m}$ above the site elevation (sea surface). To be consistent with the altimeterretrieved U10, the buoy-measured winds were converted to 10-m neutral wind using a log-profile relation assuming that the air-sea boundary layer is neutrally stable [17]. Besides wind vectors, the buoys also provide other oceanic and atmospheric information, including the SWH, Mean Wave 
Period (MWP), Dominant Wave Period (DWP), Mean Wave Direction (MWD), SST, SSAT, and atmospheric pressure. The SWH, MWP, and DWP are estimated from the one-dimensional wave spectra while the MWD is from the directional wave spectra reconstructed by the four directional Fourier coefficients $[18,19]$. Detailed information about the buoy data and all the aforementioned parameters are available from the NDBC website (www.ndbc.noaa.gov).

\subsection{Collocation}

The multiplatform altimeter dataset was collocated with the NDBC buoy dataset to make comparisons between them. The spatial and temporal windows for the collocation were set to $50 \mathrm{~km}$ and $30 \mathrm{~min}$, respectively, which is a widely used criterion in previous studies (e.g., [3,17]). When several retrievals could be collocated with a buoy in the same satellite pass, only the one that is the nearest to the buoy was used. A total of 33,404 collocations were obtained using this criterion, and the number of collocations for each buoy is shown in Figure 1.

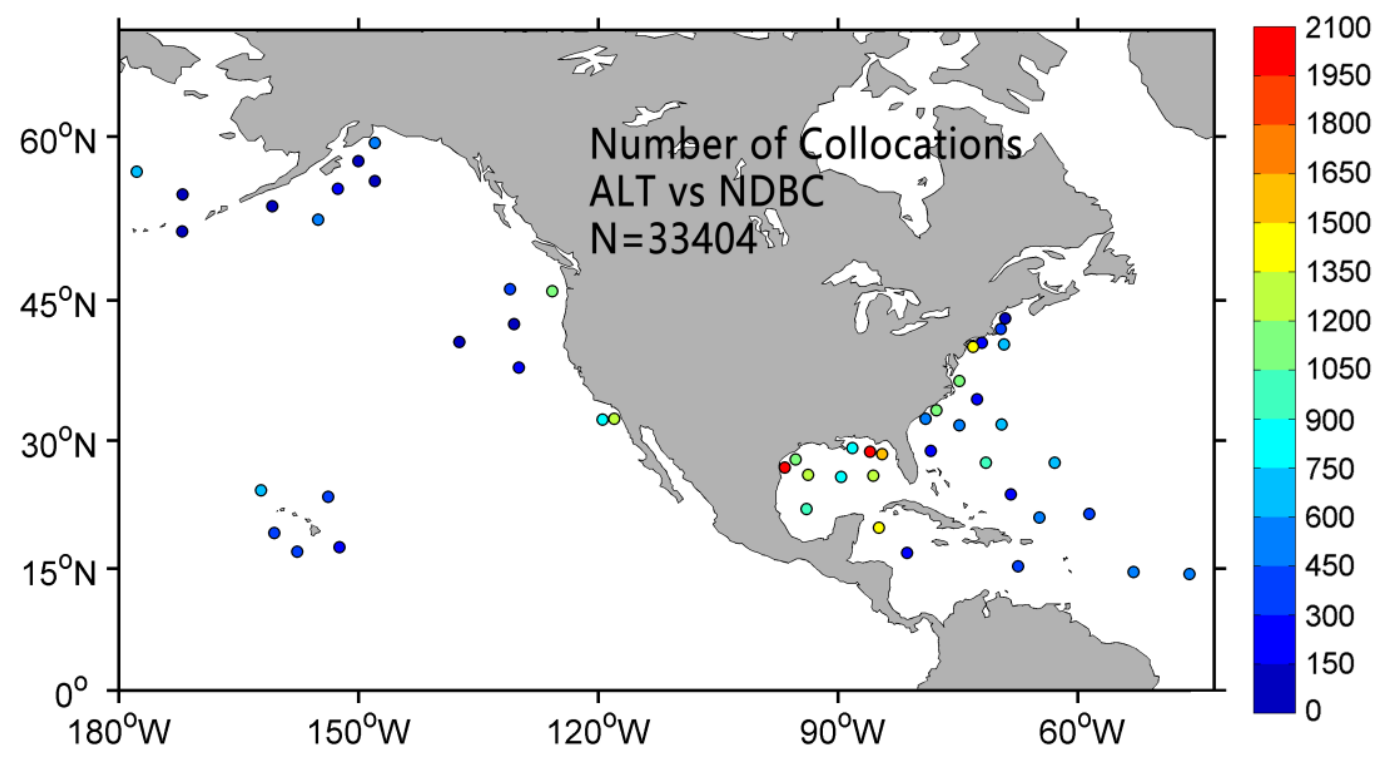

Figure 1. The locations of NDBC buoys used in this study. The color of the dots indicates the number of collocations at each site.

\section{Correlation of the U10 Error}

A comparison between the multiplatform altimeter U10 and buoy U10 is shown as a scatter plot in Figure 2a. Some standard error metrics, including the root-mean-square error (RMSE), correlation coefficient (CC), and bias, are used to evaluate the data. Although the altimeter U10 has been jointly calibrated against each other and against the buoy measurements, Figure 2a shows that there is a small bias of $0.14 \mathrm{~m} / \mathrm{s}$ and the least-square regression line does not perfectly lie on $y=x$. This is probably because more buoy data were used in the calibration of the dataset in [3] and there could be systematic error among different buoys. The RMSE of the altimeter U10 retrievals is $1.45 \mathrm{~m} / \mathrm{s}$, and the CC between the two datasets is 0.898 . After re-calibrating the U10 data using the regression line (Figure $2 \mathrm{~b}$ ), the bias is eliminated and the RMSE is also slightly reduced to $1.42 \mathrm{~m} / \mathrm{s}$.

The residuals between altimeter and buoy U10 were computed for each collocation, and the CCs between the U10 residuals and the geophysical parameters measured by buoys were then computed with the results shown in Figure 3. Of course, the relation between U10 residuals and geophysical parameters can hardly be perfectly linear, but the CC is helpful to give a reference as to whether the dependency between them is statistically significant. Although the CCs are less than 0.3 for most parameters, the U10 residual is significantly correlated to all the selected oceanic and atmospheric parameters at the $99 \%$ level (two-sided t-test, which was used for the tests of CCs throughout the paper). These CCs were also computed using several single satellites, and the results were preliminarily the same. 

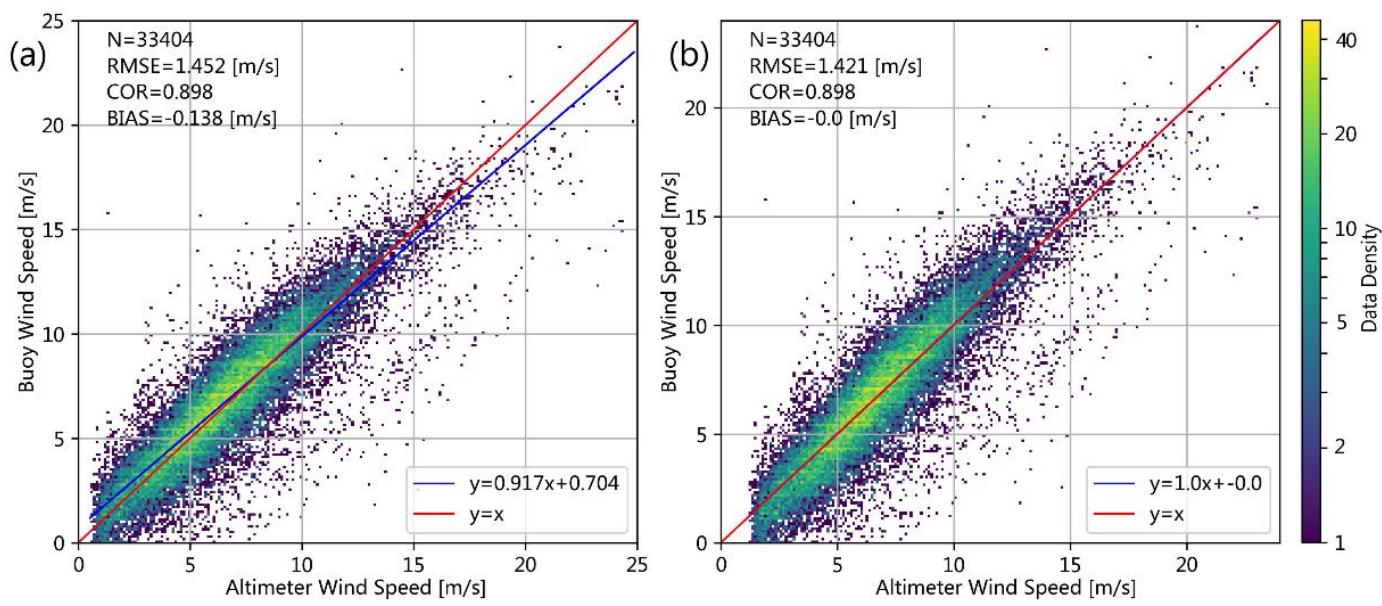

Figure 2. Scatter plots of U10 from multiplatform altimeters versus U10 from buoys: (a) the original data, and (b) the data after linear re-calibration. The blue line represents the least-square regression line. The color of dots indicates the data density.
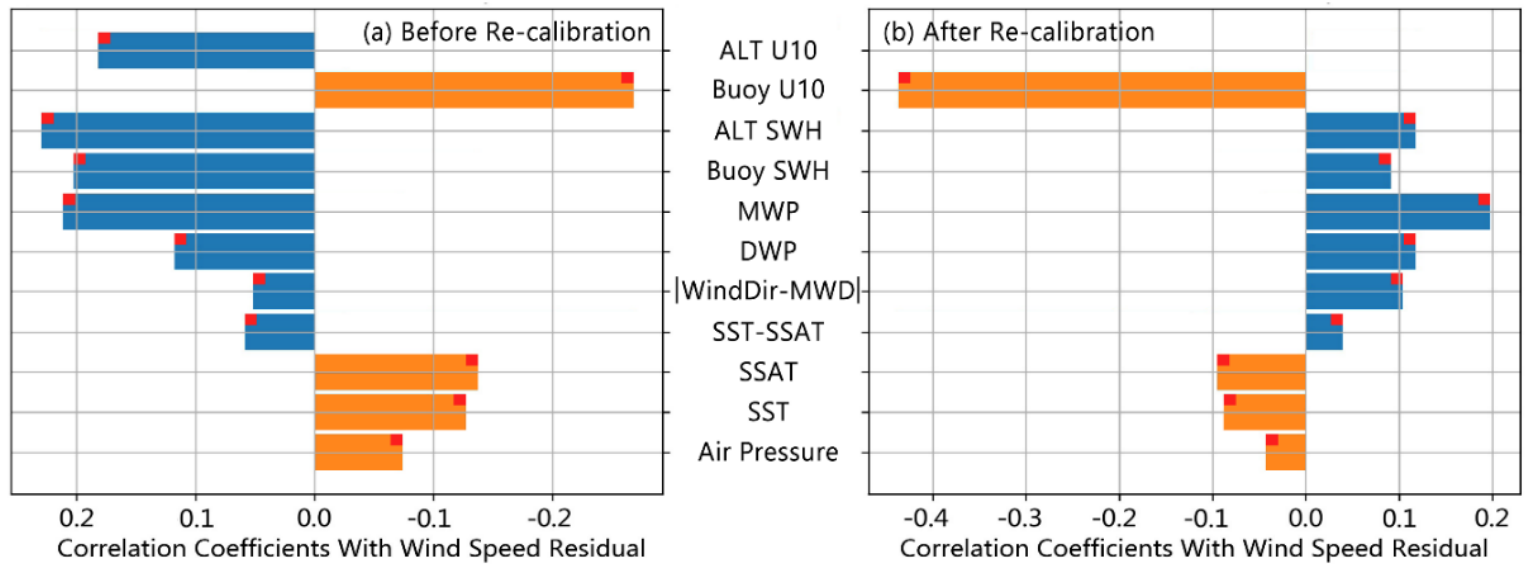

Figure 3. Correlation coefficients between U10 residuals (altimeter U10 minus buoy U10) and geophysical parameters in collocation points (a) before and (b) after linear recalibration. The red square in the corner of the bar denotes statistical significance at the $99 \%$ level.

The correlation of the U10 residuals is positive with the altimeter U10 but is negative with the buoy U10 before re-calibration. This means that altimeters tend to overestimate low wind speed and underestimate high wind speed. However, there is also an overestimation and an underestimation when the altimeter-retrieved U10 is high and low, respectively. This seems to be somehow contradictory, but this phenomenon can be clearly seen in Figures $4 \mathrm{a}$ and $4 \mathrm{~b}$, where the residuals increase with altimeter U10 but decrease with buoy U10, and larger errors occur when the buoy or altimeter U10 is high or low. Particularly, when the buoy U10 is less than $1.5 \mathrm{~m} / \mathrm{s}$, there is a systematical overestimation for $1 \sim 2 \mathrm{~m} / \mathrm{s}$ because altimeter $\mathrm{U} 10$ does not even have any records of $\mathrm{U} 10$ $<1 \mathrm{~m} / \mathrm{s}$. After linear re-calibration, the residuals are not correlated to altimeter U10 anymore (Figure $4 \mathrm{c})$, but their correlation with buoy U10 becomes higher (CC $<-0.4)$. This means that although the leastsquare linear calibration against in-situ data can reduce the overall RMSE, the systematic error that underestimates high winds and overestimates low winds might be magnified (both the slope and intercept are larger in Figure $4 \mathrm{~d}$ than Figure $4 \mathrm{~b}$, and the averaged error for no wind condition is larger than $2 \mathrm{~m} / \mathrm{s}$ in Figure $4 \mathrm{~d}$ ). Because the wind speed itself is the parameter to be retrieved, this systematic error is hard to correct. Increasing the residuals' CC with one U10 through linear transform will decrease the CC with the other one; thus, applying the regression equation of U10 residuals in Figure $4 \mathrm{~b}$ to the altimeter U10 directly will only lead to a larger RMSE and might cause negative retrieved U10 values. 

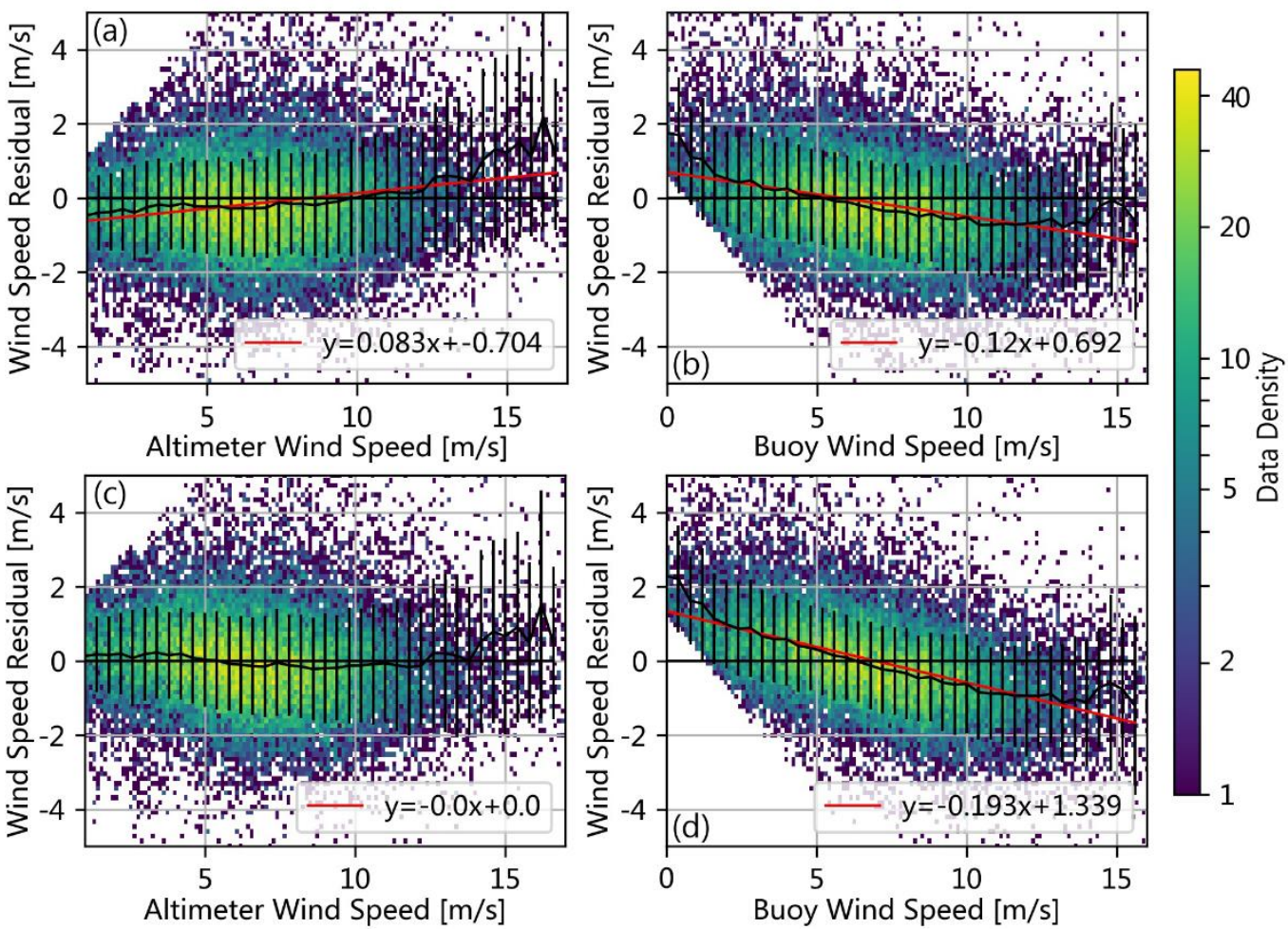

Figure 4. Scatter plots for U10 residuals relative to (a \& c) altimeter U10 and (b \& d) buoy U10, (a \& b) before and ( $c \& d$ ) after linear re-calibration. The black solid lines show the average residuals and the error bars show the corresponding standard deviations. The red lines represent least-square linear regression.

The wave parameters, especially SWH and MWP, are positively correlated to the residuals, and the SST and SSAT are negatively correlated to the residuals, which is consistent with the results of [12]. The negative correlation of the U10 residuals on SST and SSAT indicates that the change of dynamic viscosity [11] might not be important for altimeter wind speed retrieval because a higher SST corresponds to a lower viscosity, which should lead to an overestimation of the U10. The SST and SSAT might simply change the wind profiles by impacting the atmospheric instability without directly changing the SSR. Before re-calibration, the SWH has the largest CC among the selected parameters, but this value is greatly reduced after re-calibration. This is because the overall SWH is closely correlated to U10, especially when wind-sea is dominant. Figure 1 shows that more than half of the collocations are located in semi-enclosed basins where wind-sea tends to be dominant [20] so that re-calibration with U10 also reduces the error's dependence on SWH. Besides, the CCs with other oceanic and atmospheric parameters are also slightly reduced after re-calibration except for the angles between wind and waves. Table 1 shows the correlation coefficient among these parameters in the collocation dataset. Most of the values in the table are significant at the $99 \%$ level. Within expectation, the strongest correlation is the SST and SST. The three wave parameters, SWH, MWP, and DWP, also show strong correlations with each other. The parameter with the weakest overall correlation with the other parameters is the difference between SST and SSAT. The potential reason for these correlations is that most of these parameters have significant seasonality. The re-calibration using altimeter U10 slightly reduced the seasonality of the U10 residual (Figure 5), which also partially reduced the dependence of errors on other parameters. After re-calibration, the parameter with the largest CC becomes MWP which is less correlated with U10.

To further explore the relationship between the U10 residual and the aforementioned parameters, the U10 residuals are shown in Figure 6 as the functions of these parameters. Before wind re-calibration, the U10 tends to be underestimated for $\mathrm{SWH}<2 \mathrm{~m}$ and overestimated for $\mathrm{SWH}>2 \mathrm{~m}$ (Figure 6a; it is noted that the error of altimeter retrieved SWH is very small [17]). After re-calibration (Figure $6 \mathrm{~b}$ ), the underestimation in low SWH is almost eliminated and the overestimation in high 
SWH is also alleviated but still exists. Waves, especially swells, could impact the remotely sensed U10 in many ways. Besides the mechanisms mentioned in Section 1, swells can also change the surface wind profile by inducing the wave-driven winds [21]. Another important parameter to describe the wave state is the MWP which is representative of the wave's horizontal scale. However, the MWP is less dependent on U10 than the SWH so that the CC of the MWP is only slightly reduced after re-calibration. Figure $6 \mathrm{c}$ shows that the U10 tends to be underestimated for MWP $<6 \mathrm{~s}$ and overestimated for MWP $>6 \mathrm{~s}$. It is shown that sea-state bias for measuring the sea surface height is closely linked to MWP because the shape of the Stokes wave is dependent on the MWP [22]. This effect seems to also be effective for altimeter U10 retrieving. When the MWP is low, the shape of the wave profile is more like a Stokes wave so that the area of wave troughs, which is smoother and has a smaller incidence angle than wave crests, is larger, leading to an underestimation of wind speed. Because the mean residual is zero after re-calibration, the U10 retrievals in high MWP are overestimated.

Table 1. The correlation coefficients among different oceanic and atmospheric parameters in the collocation dataset.

\begin{tabular}{|c|c|c|c|c|c|c|c|c|}
\hline & $\mathbf{U 1 0}^{1}$ & SWH $^{1}$ & MWP & DWP & $|\Delta \theta|^{2}$ & $\Delta \mathbf{T}^{3}$ & SST & SSAT \\
\hline SWH & $0.68^{*}$ & & & & & & & \\
\hline MWP & $0.11^{*}$ & $0.70^{*}$ & & & & & & \\
\hline DWP & 0.03 & $0.49^{*}$ & $0.79^{*}$ & & & & & \\
\hline $\mathbf{\Delta} \boldsymbol{\theta} \mid$ & $-0.27^{*}$ & $-0.10^{*}$ & $0.16^{*}$ & $0.29^{*}$ & & & & \\
\hline$\Delta \mathbf{T}$ & $0.16^{*}$ & $0.07^{*}$ & -0.03 & -0.04 & 0.02 & & & \\
\hline SST & $-0.23^{*}$ & $-0.36^{*}$ & $-0.34^{*}$ & $-0.31^{*}$ & $-0.12^{*}$ & -0.06 & & \\
\hline SSAT & $-0.26^{*}$ & $-0.36^{*}$ & $-0.31^{*}$ & $-0.28^{*}$ & $-0.11^{*}$ & $-0.33^{*}$ & $0.96^{*}$ & \\
\hline AP & $-0.25^{*}$ & $-0.30^{*}$ & $-0.13^{*}$ & $-0.06^{*}$ & 0.01 & $0.19^{*}$ & $0.15^{*}$ & $0.09^{*}$ \\
\hline
\end{tabular}

${ }^{1}$ The U10 and SWH data are from altimeters.

2 The angle between wind direction and MWP.

${ }^{3}$ The difference between SST and SSAT.

${ }^{4}$ The "**" represents statistical significance at the $99 \%$ level.
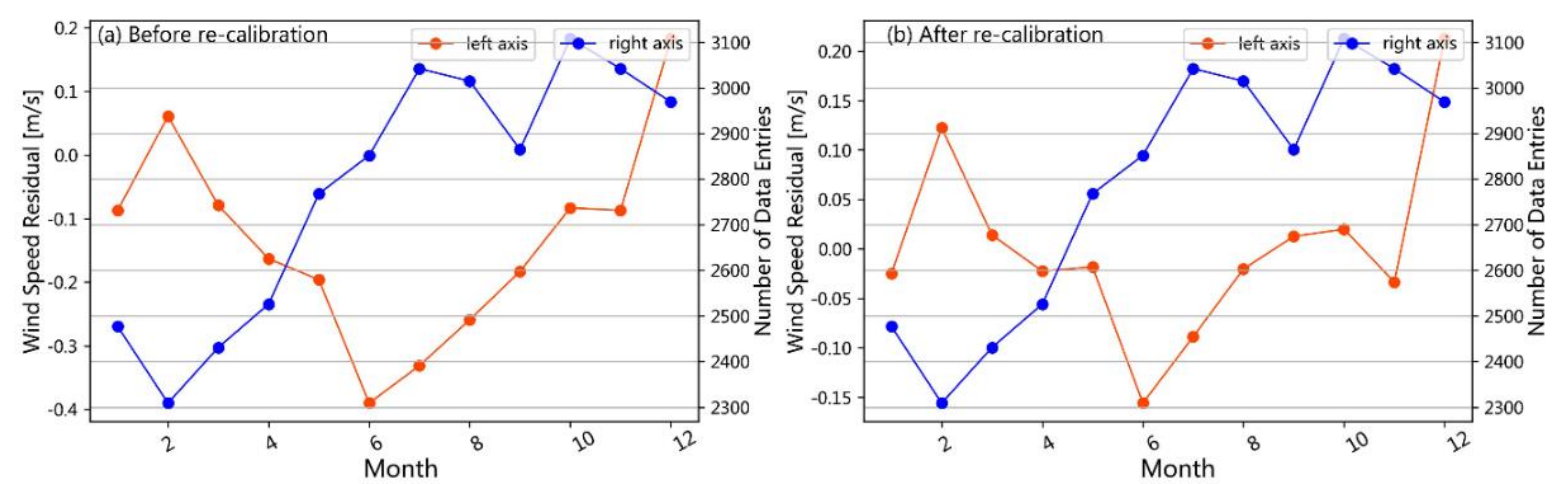

Figure 5. Averaged U10 residuals as a function of calendar month (red lines, left axis) and the numbers of collocations as a function of calendar month (blue lines, right axis), (a) before and (b) after linear re-calibration. It is noted that the left axes of the two subplots are different.

Compared to the SWH and MWP, the impacts of the other parameters are small. The scatter plots for residuals relative to the air pressure, the angle between wind and wave, and the SST are shown in Figures 6d, 6e, and 6f, respectively. Although the correlation of air pressure is statistically significant, this is primarily due to the slight overestimation in a few low-pressure conditions. In the range between 1000 to $1030 \mathrm{hPa}$, the mean of the U10 residuals is not significantly different from zero 
(two-sided t-test at the 95\% level). The dependence of the U10 residuals on the difference between wind-wave angles is also close to a linear one, which might be due to the Stokes drifts-induced relative motion of the air-sea interface that can impact the relative wind speed sensed by space-borne sensors. This dependence induces an underestimation of $\sim 0.15 \mathrm{~m} / \mathrm{s}$ when the wind direction is in line with the mean wave direction, which is common in the wind-sea-dominated conditions. There is an underestimation of $\mathrm{U} 10(\sim 0.3 \mathrm{~m} / \mathrm{s})$ for the SST ranging from 12 to $30^{\circ} \mathrm{C}$ and an overestimation $(\sim 0.5$ $\mathrm{m} / \mathrm{s}$ ) for the SST lower than $12^{\circ} \mathrm{C}$ before re-calibration (not shown), but the mean residual from 12 to $30{ }^{\circ} \mathrm{C}$ is not significantly different from zero after re-calibration (two-sided t-test at the 95\% level) while there is still a systematic overestimation for $\sim 0.4 \mathrm{~m} / \mathrm{s}$ when SST is lower than $12{ }^{\circ} \mathrm{C}$. The dependence on the SSAT is generally the same as on the SST (not shown). The low SST data are mainly from the nine buoys along the Alaska coast, thus, this error might simply be geographical errors, although the SST affecting U10 retrievals by impacting the atmospheric instability is also a plausible explanation.
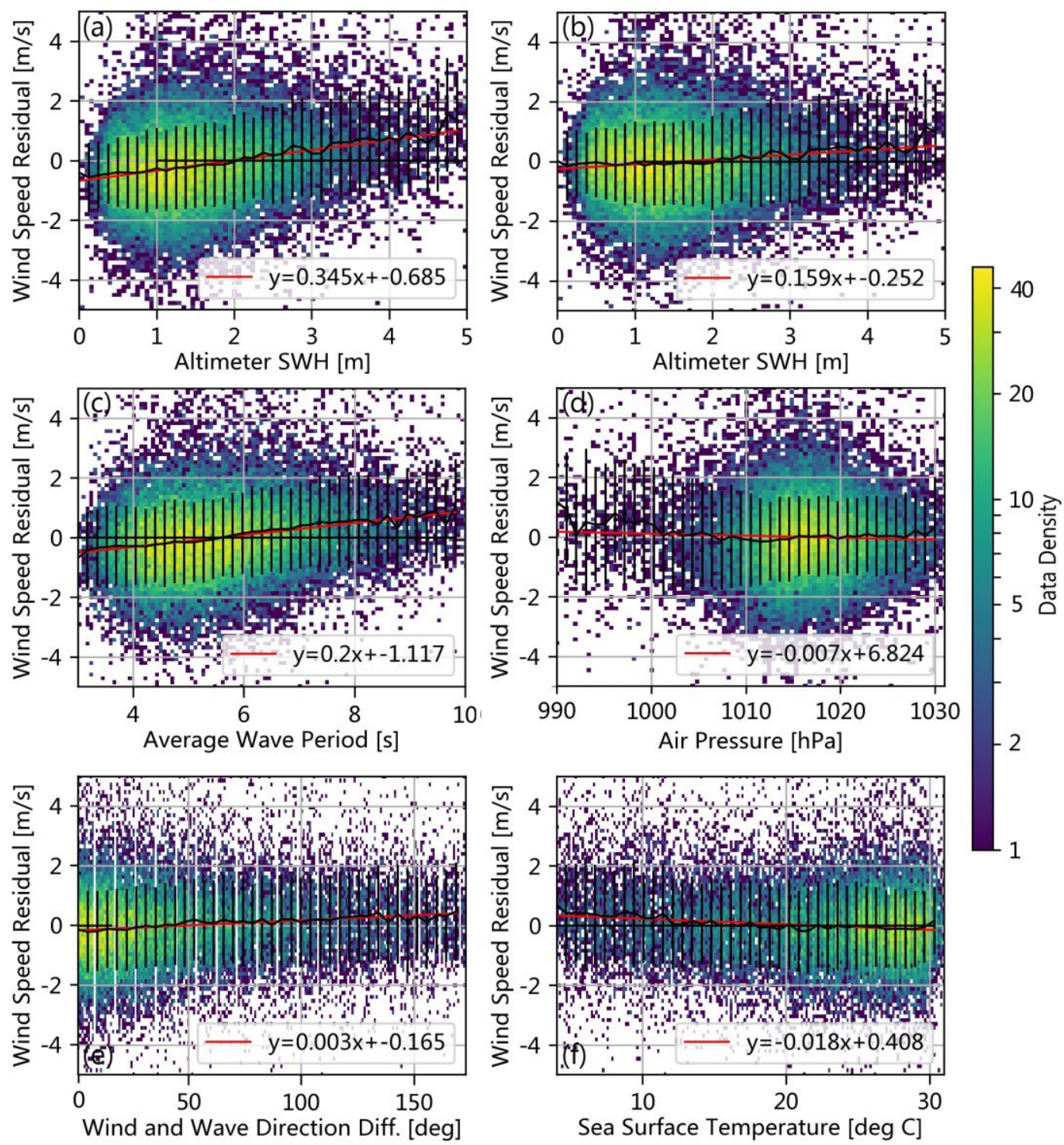

Figure 6. Scatter plots for U10 residuals relative to (a \& b) SWH, (c) MWP, (d) air pressure, (e) angles between wind direction and MWD, and (f) SST. All the results are after linear re-calibration except for subplot (a). The black solid lines show the average residuals and the error bars show the corresponding standard deviations. The red lines represent least-square linear regression.

\section{Retrieval Improvement}


Statistically significant dependence is found between the error of altimeter wind speed retrievals and some oceanic and atmospheric parameters using altimeter-buoy collocations. Although some reasonable explanations can be given on mechanisms of the dependence on different parameters, it might be difficult to directly prove these mechanisms using the collocation data at this stage, and the inter-dependence among different geophysical quantities makes this task more difficult. Meanwhile, quantitively knowing these dependences can be useful for further reducing the error of altimeter U10 retrievals. Here, a trial was made to establish a nonparametric model for correcting altimeter U10 retrievals by considering some of the parameters. A simple three-layer neural network (including an input layer, a hidden layer, and an output layer) was employed here. The neural network was realized by the MATLAB Deep Learning Toolbox ${ }^{\mathrm{TM}}$. The dataset of altimeter-buoy collocations was randomly divided into two parts, one for model training (23,404 records) and the other for model validation (10,000 records). The number of neurons in the hidden layer was set to 21 after some tests.

From Figures 4 and 6, it can be seen that the dependencies between the U10 residuals and oceanic/atmospheric parameters do not have strong nonlinearity. Therefore, the CC can be a good metric to quantify the dependence. The correction model was trained using an asymptotic strategy as follows:

- First, a correction model was trained using only the original altimeter U10 as the input parameter.

- Second, the RMSE of correction model output and the CCs between the U10 residuals and the aforementioned parameters were computed

- Third, the parameter with the highest CC was found and a new correction model was trained by taking this parameter into account.

- The $2^{\text {nd }}$ and $3^{\text {rd }}$ steps are repeated until the RMSE of the new correction model was not significantly lower than the RMSE of the previous one.

Using this strategy, the first four parameters that need to be taken into account are found to be the MWP, SWH, DWP, and SST-SSAT difference, respectively. The corresponding scatter plots are shown in Figure 7. Compared to Figure 2, the RMSE is significantly reduced from $1.42 \mathrm{~m} / \mathrm{s}$ to 1.35 $\mathrm{m} / \mathrm{s}(1.35 \mathrm{~m} / \mathrm{s}$ for the training dataset) and the CC increases from 0.898 to 0.905 ( 0.908 for the training dataset) after considering the dependence on MWP (Figure 7a). The RMSE further decreases to 1.26 $\mathrm{m} / \mathrm{s}(1.24 \mathrm{~m} / \mathrm{s}$ for the training dataset) with CC increasing to 0.918 ( 0.921 for the training dataset) after considering the effect of SWH (Figure 7b). Then, after considering DWP (Figure 7c), there is still a slight improvement on both RMSE $(1.26 \mathrm{~m} / \mathrm{s}$ to $1.23 \mathrm{~m} / \mathrm{s})$ and CC $(0.918$ to 0.922$)$, but the errors are not further reduced with adding the fourth parameter (the SST-SSAT difference, which is not shown).

The three most effective parameters to improve wind speed retrievals are all surface wave parameters, which indicates that the impact of ocean waves is much more important than the impact of other ocean and atmospheric parameters. In the three wave parameters, the SWH is the one that has been widely used for wind speed retrieval in previous studies (e.g., [10,12,15]) as it is directly available from the altimeter. In addition, algorithms have been presented to use U10, SWH, and MWP to correct sea-state bias in measuring sea surface height (e.g., [22]). This study shows that MWP is also an effective parameter in improving the accuracy of altimeter U10 retrieval. Some algorithms have been established on retrieving wave period information from altimeter data (e.g., [23-26]). However, the algorithms in most of these studies (e.g.,[23-25]) are established based on the empirical relation assuming that the wave period is a function of $\mathrm{U} 10$ (or RCS) and SWH, which is not very helpful for improving U10 retrievals (as it does not increase the input information). The idea of using spatial derivate of SWH presented in [26] is promising for providing an independent input, but this algorithm is not yet ready for operational use. Another source of independent MWP information is the numerical wave model. Contemporary numerical wave models can have a fairly good performance on simulating many wave parameters including MWP (e.g.,[27]), which can be used in this correction model.

Figure 8a shows the U10 residual as a function of DWP after being corrected using MWP and SWH. The mean U10 residual is not statistically different from zero when the DWP is shorter than $12 \mathrm{~s}$, but there is an underestimation of the altimeter U10 when the DWP is longer than 12s which means the sea is swell-dominated. When the sea is either overwhelmingly dominated by wind-seas 
or swells, the wave spectrum can be regarded as a unimodal one so that the value of MWP should be close to DWP so that the DWP becomes a redundant parameter. When the sea state is bimodal or multimodal, the DWP can be very different from the MWP. However, it is noted that including DWP is still insufficient to describe this complex sea-state, and DWP is not a stable wave parameter when spectral densities of the two highest peaks are close to each other. Therefore, the improvement of U10 retrieval after considering DWP is marginal. Due to these reasons, a correction model using simply U10, MWP, and SWH seems to be more practical.
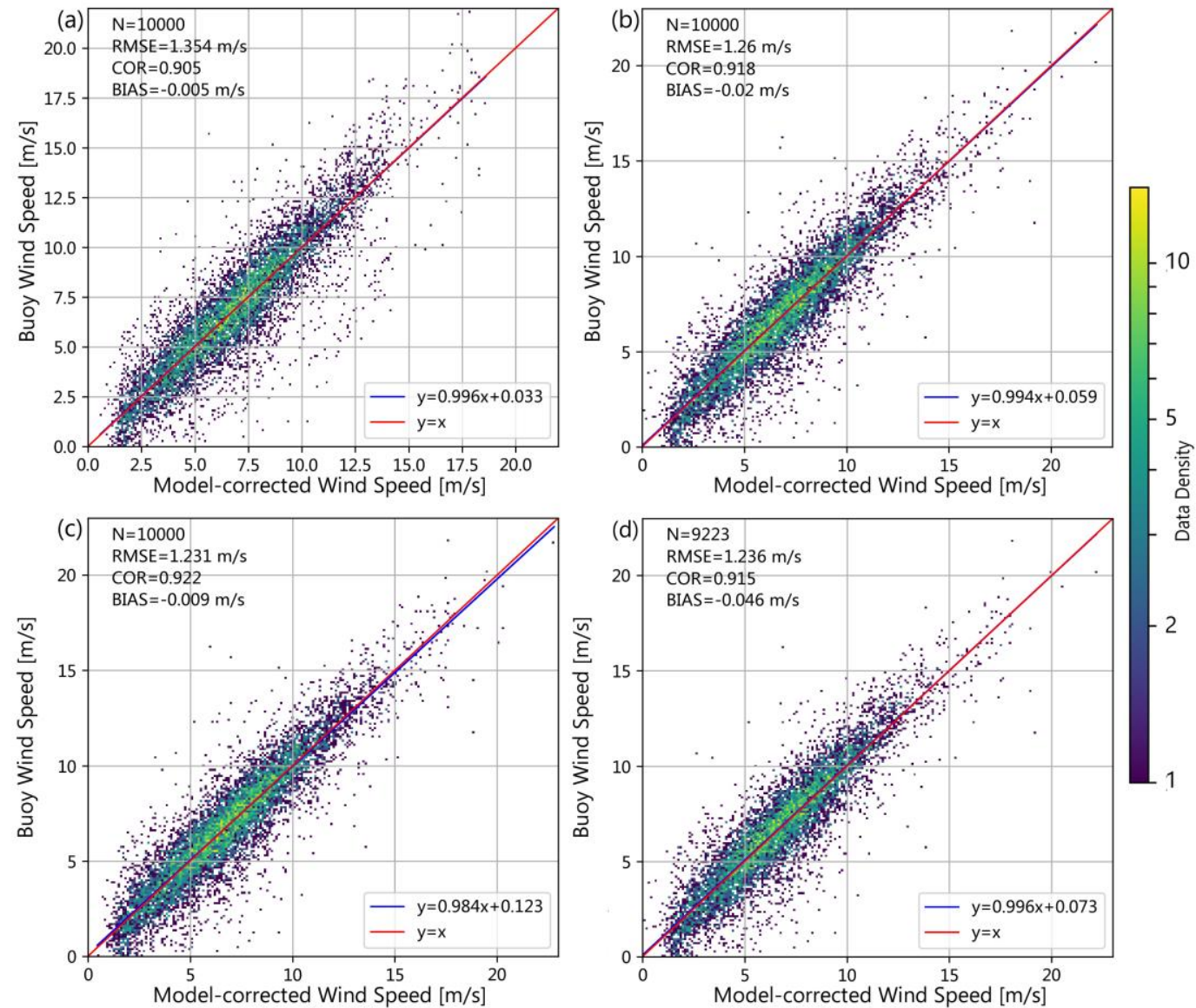

Figure 7. The scatter plots of U10 from buoy versus U10 multiplatform altimeters corrected by models taking different parameters into account: (a) U10 and MWP, (b) U10, MWP, and SWH, (c) U10, MWP, SWH, and DWP. Subplot (d) is the same as (b), but the points with SST-SSAT difference greater than $4^{\circ} \mathrm{C}$ are excluded. The blue lines represent the least-square regression lines. The color of dots indicates the data density.

It is noted that the U10 from the buoy was obtained using a logarithmic wind profile under the assumption of neutral atmospheric stability. Therefore, the variation of atmospheric stability can lead to deviation from this profile and then lead to an error of the buoy U10. Previous studies found the dependencies of remotely sensed U10 residuals on air pressure and SST/SSAT (e.g., [11,12]), and attributed these dependencies to the impact of atmospheric instability and dynamic viscosity. However, after the correction using MWP and SWH, the U10 residuals' CCs with air pressure and wind-wave angle becomes insignificant (at 95\% level) and error dependencies on SST and SSAT become much less than the dependence on their SST-SSAT difference (not shown). This indicates that some of these dependences might simply be due to the universal inter-correlation of geophysical parameters as they all have seasonality. Even if the impacts of air pressure, SST, and SSAT are through some physical processes, such as changing dynamic viscosity, they can also be largely corrected by using some wind and wave parameters. 

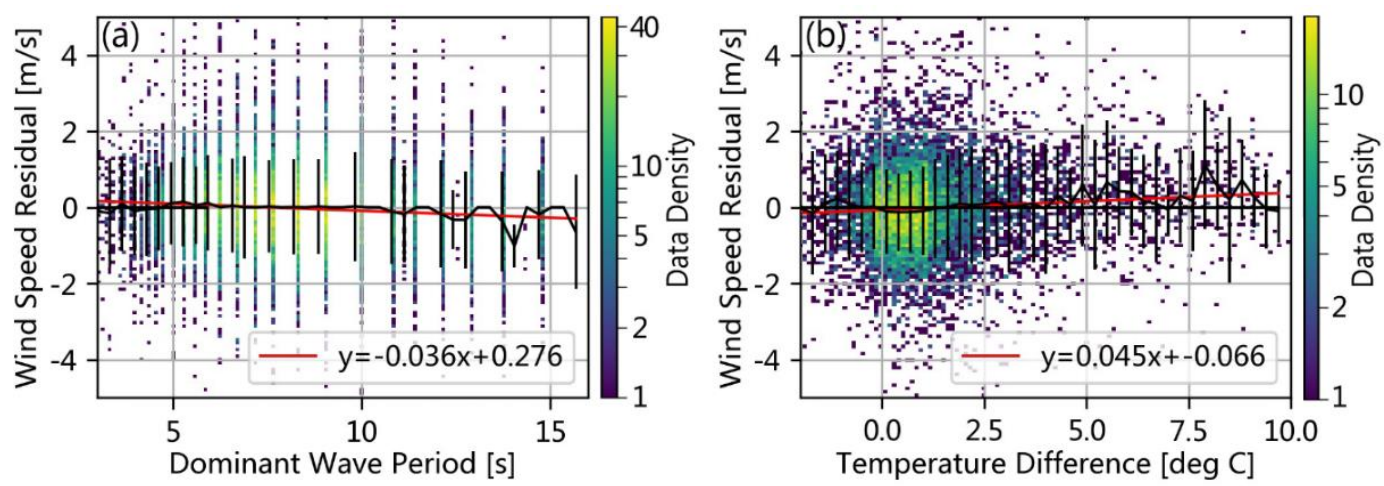

Figure 8. Scatter plots for U10 residuals relative to (a) DWP and (b) SST-SSAT difference after correction using U10, SWH, and MWP. The black solid lines show the average residuals and the error bars show the corresponding standard deviations. The red lines represent least-square linear regression.

Meanwhile, this does not mean that the atmospheric instability is not important for the Cal/Val of remotely sensed U10. The most direct indicator for atmospheric stability among the selected parameters is the SST-SSAT difference, which is also the parameter relatively independent of other ones (Table 1). The wave boundary layer will be in an unstable state when the sea is warmer than the air, and vice versa. Although considering the SST-SSAT difference does not help for a better algorithm, the correlation of the U10 residuals with the SST-SSAT difference is significant $(0.08$, which significant at the $99 \%$ level) for the results in Figures $7 \mathrm{~b}$ and $7 \mathrm{c}$. To further explore this dependence, the U10 residual as a function of SST-SSAT difference is shown in Figure 8b. The air-sea temperature difference for around $90 \%$ of the data is within $\pm 2.5^{\circ} \mathrm{C}$, and the bias of U10 is negligible in this range. However, when the difference becomes higher, which means the sea is warmer than the air above (unstable condition), the altimeter systematically overestimate the $\mathrm{U} 10$ for about $0.5 \mathrm{~m} / \mathrm{s}$. In this case, the buoy $\mathrm{U} 10$ obtained from the logarithmic profile of neutral stability itself is overestimated, and the buoy data are not a good U10 reference anymore because it is difficult to determine whether this error comes from the remote sensors or the buoys. Therefore, we suggest that buoy U10 with high air-sea temperature difference should not be used for the Cal/Val and error analysis of remotely sensed U10. After excluding the "bad-quality" buoy data with an SST-SSAT difference greater than $4^{\circ} \mathrm{C}(777$ points), it is found that the altimeter U10 can have a slightly better comparison with the buoy U10 with an RMSE of $1.24 \mathrm{~m} / \mathrm{s}$ (Figure 7d).

\section{Conclusions}

Spaceborne altimeters can provide nadir wind speed information because the RCS is strongly dependent on U10. Although the U10 is the most important impact factor of the RCS, the RCS can also have some secondary response to other oceanic and atmospheric parameters, such as the SWH, MWP, SST, and SSAT, which has been confirmed by previous studies using scatterometer and SAR data (e.g., [10-12] ). In this study, the impact of these secondary responses on altimeter U10 retrievals was analyzed using the colocations between ten satellite altimeters and 52 NDBC buoys. These collocations allow an assessment on the performance of altimeter U10 retrievals and a detailed description of the dependency between the altimeter U10 residuals and some oceanic and atmospheric parameters obtained by the buoys.

After the joint calibration [3], the U10 from the multiplatform altimeters generally show a good agreement with the buoy U10 with an RMSE of $1.45 \mathrm{~m} / \mathrm{s}$. Meanwhile, the U10 residuals are found to be weakly but significantly correlated to many oceanic and atmospheric parameters, which means some of the errors of the altimeter U10 retrievals are systematic and can be corrected using these parameters. Because oceanic and atmospheric parameters are usually inter-correlated, an asymptotic strategy is used to establish a correction model of altimeter U10. The results indicated that the altimeter U10 retrievals are the most significantly impacted by the SWH and MWP. A reduced RMSE of $1.24 \mathrm{~m} / \mathrm{s}$ can be obtained using the correction model taking ocean waves into account. The impacts 
of many other parameters, such as air pressure, SST, SSAT, become insignificant after the wave correction. Using the dependency between the SST-SSAT difference and residuals of corrected U10, it is also found that the atmospheric instability can lead to some errors on the buoy extrapolated U10. Thus, another conclusion that can be drawn is that the cases with large SST-SSAT differences should be excluded in the comparison between remote sensed and in-situ wind speed.

In this study, the correction was based on the collocation between buoys and ten satellites for a better statistical significance. The altimeter U10 which has been jointly calibrated was used as an input term of the correction model. Operational altimeter missions may use a different GMF than the multiplatform satellite dataset. Therefore, the correction model derived from the collocations in this study may not be directly applicable to them. However, the same idea can also be used in establishing operational altimeter GMFs with $\mathrm{Ku} / \mathrm{Ka}$-band RCS, SWH, and MWP (from numerical wave forecast/hindcast), which can be a direction for the future study. Some altimeters, such as TOPEX, Jason-1/2/3, HY-2A, and Sentinel-3A, use the Ku-and-C dual-frequency setup, which can also be used to improve U10 accuracy [28]. A recent study showed that the difference between the RCS of the two bands is related to ocean wave information [29]. Therefore, further study can also be made to investigate whether this wave correction is redundant for the dual-frequency U10-retrieving approach.

Author Contributions: $\mathrm{H}$. J. presented the idea and wrote the original draft; H. Z. processed the data and plotted most of the figures under the guidance of H. J.; L. M contributed to the discussion; all authors have read and agreed to the published version of the manuscript.

Funding: This work was jointly supported by the National Natural Science Foundation of China (Grant No. 41806010) and the Discipline Layout Project for Basic Research of Shenzhen Science and Technology Innovation Committee (Grant No. JCYJ20170810103011913).

Acknowledgments: The authors would like to thank Prof. Ian Young and Dr. Agustinus Ribal for sharing the jointly-calibrated altimeter data. The buoy data are downloaded from the NDBC website (https://www.ndbc.noaa.gov/).

Conflicts of Interest: The authors declare no conflict of interest.

\section{References}

1. Young, I.R.; Sanina, E.; Babanin, A. V Calibration and Cross Validation of a Global Wind and Wave Database of Altimeter, Radiometer, and Scatterometer Measurements. J. Atmos. Ocean. Technol. 2017, 34, 1285-1306.

2. Ribal, A.; Young, I.R. Calibration and cross-validation of global ocean wind speed based on scatterometer observations. J. Atmos. Ocean. Technol. 2020, in press.

3. Ribal, A.; Young, I.R. 33 years of globally calibrated wave height and wind speed data based on altimeter observations. Sci. Data 2019, 6, 77.

4. Zhao, D.; Li, S.; Song, C. The comparison of altimeter retrieval algorithms of the wind speed and the wave period. Acta Oceanol. Sin. 2012, 31, 1-9.

5. Young, I.R.; Ribal, A. Multiplatform evaluation of global trends in wind speed and wave height. Science 2019, 9527, 1-10.

6. Chen, G.; Chapron, B.; Ezraty, R.; Vandemark, D. A global view of swell and wind sea climate in the ocean by satellite altimeter and scatterometer. J. Atmos. Ocean. Technol. 2002, 19, 1849-1859.

7. Jiang, H.; Chen, G. A global view on the swell and wind sea climate by the Jason-1 mission: A revisit. J. Atmos. Ocean. Technol. 2013, 30, 1833-1841.

8. Hoffman, R.N.; Louis, J.-F. The influence of atmospheric stratification on scatterometer winds. J. Geophys. Res. Ocean. 1990, 95, 9723-9730.

9. Plagge, A.M.; Vandemark, D.; Chapron, B. Examining the Impact of Surface Currents on Satellite Scatterometer and Altimeter Ocean Winds. J. Atmos. Ocean. Technol. 2012, 29, 1776-1793.

10. Li, H.; Mouche, A.; Stopa, J.E. Impact of Sea State on Wind Retrieval From Sentinel-1 Wave Mode Data. IEEE J. Sel. Top. Appl. Earth Obs. Remote Sens. 2019, 12, 559-566.

11. Wang, Z.; Stoffelen, A.; Fois, F.; Verhoef, A.; Zhao, C.; Lin, M.; Chen, G. SST Dependence of Ku- and CBand Backscatter Measurements. IEEE J. Sel. Top. Appl. Earth Obs. Remote Sens. 2017, 10, 2135-2146.

12. Stopa, J.E.; Mouche, A.A.; Chapron, B.; Collard, F. Sea State Impacts on Wind Speed Retrievals From C-Band Radars. IEEE J. Sel. Top. Appl. Earth Obs. Remote Sens. 2017, 10, 2147-2155. 
13. Wang, Z.; Stoffelen, A.; Zhao, C.; Vogelzang, J.; Verhoef, A.; Verspeek, J.; Lin, M.; Chen, G. An SSTdependent Ku-band geophysical model function for RapidScat. J. Geophys. Res. Ocean. 2017, 122, 34613480 .

14. Monaldo, F.; Dobson, E. On using significant wave height and radar cross section to improve radar altimeter measurements of wind speed. J. Geophys. Res. Ocean. 1989, 94, 12699-12701.

15. Gourrion, J.; Vandemark, D.; Bailey, S.; Chapron, B.; Gommenginger, G.P.; Challenor, P.G.; Srokosz, M.A. A Two-Parameter Wind Speed Algorithm for Ku-Band Altimeters. J. Atmos. Ocean. Technol. 2002, 19, 2030-2048.

16. Hausman, J.; Zlotnicki, V. Sea State Bias in Radar Altimetry Revisited. Mar. Geod. 2010, 33, $336-347$.

17. Zieger, S.; Vinoth, J.; Young, I.R. Joint calibration of multiplatform altimeter measurements of wind speed and wave height over the past 20 Years. J. Atmos. Ocean. Technol. 2009, 26, 2549-2564.

18. Steele, K.E.; Mettlach, T.R. NDBC wave data - current and planned. In Proceedings of the Ocean Wave Measurement and Analysis - Proceedings of the Second International Symposium; 1993; pp. 198-207.

19. Earle, M.D.; Steele, K.E.; Wangc, D.W.C. Use of advanced directional wave spectra analysis methods. Ocean Eng. 1999, 26, 1421-1434.

20. Qian, C.; Jiang, H.; Wang, X.; Chen, G. Climatology of Wind-Seas and Swells in the China Seas from Wave Hindcast. J. Ocean Univ. China 2020, 19, 90-100.

21. Semedo, A.; Saetra, Ø.; Rutgersson, A.; Kahma, K.K.; Pettersson, H. Wave-Induced Wind in the Marine Boundary Layer. J. Atmos. Sci. 2009, 66, 2256-2271.

22. Jiang, M.; Xu, K.; Liu, Y.; Wang, L. Estimating the Sea State Bias of Jason-2 Altimeter From Crossover Differences by Using a Three-Dimensional Nonparametric Model. IEEE J. Sel. Top. Appl. Earth Obs. Remote Sens. 2016, 9, 5023-5043.

23. Mackay, E.B.L.; Retzler, C.H.; Challenor, P.G.; Gommenginger, C.P. A parametric model for ocean wave period from Ku band altimeter data. J. Geophys. Res. Ocean. 2008, 113.

24. Miao, H.; Ren, H.; Zhou, X.; Wang, G.; Zhang, J. Study on altimeter-based inversion model of mean wave period. J. Appl. Remote Sens. 2012, 6, 1-8.

25. QUILFEN, Y.; CHAPRON, B.; COLLARD, F.; SERRE, M. Calibration/Validation of an Altimeter Wave Period Model and Application to TOPEX/Poseidon and Jason-1 Altimeters. Mar. Geod. 2004, 27, 535549.

26. Badulin, S.I. A physical model of sea wave period from altimeter data. J. Geophys. Res. Ocean. 2014, 119, 856-869.

27. Liu, Q.; Rogers, W.E.; Babanin, A. V; Young, I.R.; Romero, L.; Zieger, S.; Qiao, F.; Guan, C. Observation-Based Source Terms in the Third-Generation Wave Model WAVEWATCH III: Updates and Verification. J. Phys. Oceanogr. 2018, 49, 489-517.

28. Chen, G.; Chapron, B.; Ezraty, R.; Vandemark, D. A dual-frequency approach for retrieving sea surface wind speed from TOPEX altimetry. J. Geophys. Res. Ocean. 2002, 107, 10-19.

29. Li, S.; Shen, H.; Hou, Y.; He, Y.; Bi, F. Sea surface wind speed and sea state retrievals from dualfrequency altimeter and its preliminary application in global view of wind-sea and swell distributions. Int. J. Remote Sens. 2018, 39, 3076-3093. 